\title{
Vaccination with epimastigotes of different strains of Trypanosoma rangeli protects mice against Trypanosoma cruzi infection
}

\author{
Beatriz Basso/ $/{ }^{+}$, Edgardo Moretti/ ${ }^{1}$, Ricardo Fretes ${ }^{2}$ \\ Cátedra de Pediatría y Neonatología, ${ }^{2}$ Cátedra de Histología, Facultad de Ciencias Médicas, Universidad Nacional de Córdoba, \\ Güemes 383, B. General Paz 5000, Córdoba, Argentina ${ }^{1}$ Servicio Nacional de Chagas, Córdoba, Argentina
}

\begin{abstract}
In our laboratory, we have developed a model of vaccination in mice with Trypanosoma rangeli, a non-pathogenic parasite that shares many antigens with Trypanosoma cruzi. The vaccinated mice were protected against infection with virulent $\mathrm{T}$. cruzi. The goal of the present work was to study the protective activity of strains of $\mathrm{T}$. rangeli of different origin, with the aim of analysing whether this protective capacity is a common feature of T. rangeli. BALB/c mice were vaccinated with live or fixed epimastigotes of two T. rangeli strains, Choachi and SC-58. Vaccinated (VM) and control mice (CM) were infected with virulent T. cruzi, Tulahuen strain. The results showed that the levels of parasitemia of VM, vaccinated with the two strains of $\mathrm{T}$. rangeli were significantly lower than those developed in $C M$. The survival rate of VM was higher than that CM. Histological studies revealed many amastigote nests and severe inflammatory infiltrates in the heart and skeletal muscles of CM, whereas in the VM only moderate lymphomonocytic infiltrates were detected. Altogether, the results of the present work as well as previous studies show that the antigens involved in the protection induced by $\mathrm{T}$. rangeli are expressed in different strains of this parasite. These findings could prove useful in vaccine preparation.
\end{abstract}

Key words: vaccination - Chagas disease - Trypanosoma rangeli - Trypanosoma cruzi

Infection with Trypanosoma cruzi, the protozoan agent of Chagas disease, affects nearly 16 million people, and 75-90 million people are exposed in Latin America (Coura 2007). In several Latin American countries, there is another trypanosome, $T$. rangeli, which can infect humans but is non-pathogenic (Guhl \& Vallejo 2003). Both parasites share endemic areas and vectors, and the two have a strong antigenic relationship (Basso et al. 1987, Stevens et al. 1999).

As with many other parasitic diseases, there is no effective vaccine for humans yet available, despite numerous studies performed with different antigenic materials. Approaches ranging from subcellular fractions to recombinant antigens and even plasmid DNA-encoding antigens of T. cruzi have been used (Taibi et al. 1995, Costa et al. 1998, Wrightsman \& Manning 2000, Planelles et al. 2001, Garg \& Tarleton 2002). In our laboratory, we have developed a model of vaccination for mice with $T$. rangeli, in which mice were protected by fixed epimastigotes of a Colombian strain of T. rangeli. The immunization induced a strong reduction in parasitemia and mortality levels when compared with nonvaccinated infected mice (Basso et al. 1991).

Financial support: Area of Science and Technology, Universidad Nacional de Córdoba (SECYT-UNC), Servicio Nacional de Chagas + Corresponding author: ebi@fcm.unc.edu.ar

Received: 4 March 2008

Accepted: 12 June 2008
The aim of this work was to analyze the evolution of $T$. cruzi infection in mice that were previously vaccinated with live and fixed epimastigotes of two strains of T. rangeli of different geographical origins, to determine if the protection against $T$. cruzi infection is a common feature across different $T$. rangeli isolates.

\section{MATERIALS AND METHODS}

Animals - Balb/c mice were used and maintained in our laboratory under standard conditions

Parasites - The Tulahuen strain of T. cruzi was maintained through weekly subcutaneous inoculations of Balb/c albino mice. Bloodstream trypomastigotes used for challenging inoculations were obtained by cardiac puncture on day 14 post infection (pi). The SC-58 and Choachi strains of $T$. rangeli, kindly provided by the Laboratório de Protozoologia, Univ. Fed. Sta. Catarina, Brazil, were used. The epimastigote forms were cultured in LIT monophasic medium. Parasites were harvested in the exponential phase of growth and the immunization material was prepared in two different ways: (i) live epimastigotes (L-EPI) and (ii) fixed epimastigotes (F-EPI); these were obtained as previously described (Basso et al. 1991). Briefly, parasites were washed three times in PBS and centrifuged at $10,000 \mathrm{~g}$ at $4^{\circ} \mathrm{C} / 20 \mathrm{~min}$. Then, they were fixed with glutaraldehyde $(0.1 \%)$ for $15 \mathrm{~min}$ at room temperature, following $15 \mathrm{~min}$ at $4^{\circ} \mathrm{C}$ and washed again. Just before immunization, $1 \times 10^{9}$ parasites $/ \mathrm{ml}$ were emulsified with saponin.

Vaccination and infection schedule - Groups of 7-8 Balb/c mice (3 weeks old) were vaccinated with epimastigotes of SC-58 or Choachi strain, as previously described (Introini et al. 1998, Cervetta et al. 2003). Group (a): mice were inoculated intraperitoneally with a volume 
of $0.2 \mathrm{ml}$ containing $1 \times 10^{7}$ live epimastigotes 28 days before. Group (b): mice received subcutaneous injections in the thigh of $0.2 \mathrm{ml}$ of antigenic emulsion containing $1 \times 10^{8}$ fixed epimastigotes $28,21,7$ days before. Group (c): mice received $0.2 \mathrm{ml}$ of PBS (control group).

All mice were infected at day 0 by intraperitoneal inoculation of 1,500 blood trypomastigotes of $T$. cruzi. The parasite counts were performed in a Newbauer chamber, using $0.85 \% \mathrm{NH}_{4} \mathrm{Cl}$ as diluent (Hoff 1974). The survival rates were checked daily until day 40 pi.

All experiments reported herein were conducted in compliance with the Animal Welfare Act and in accordance with the principles set forth in ILAR (1996).

Histological studies - Mice from all experimental groups were sacrificed via ether anaesthesia. The heart, spleen, and quadriceps skeletal muscle were immediately removed, fixed in buffered $10 \%$ formalin $(\mathrm{pH} 7.0)$ and embedded in paraffin. Sections, $5 \mu \mathrm{m}$ thick, of each organ were collected at a systematic distance of 10 sections apart. A total of $300 \mu \mathrm{m}$ per organ were analyzed. All sections were stained with hematoxylin and eosin. At least 25 areas from each section were checked for parasites and histopathology under a 40X objective in a blind study.

Statistical analysis - The experimental data were analysed using Student's $t$ Test. All the results were considered statistically significant at a level of $p<0.05$.

\section{RESULTS}

Parasitemia - Fig. 1 shows the results of a representative experiment performed in animals vaccinated with the L-EPI or F-EPI $T$. rangeli Choachi strain and in nonvaccinated mice (control group), where all of the groups were infected with T. cruzi. As can be seen, by the 10 th
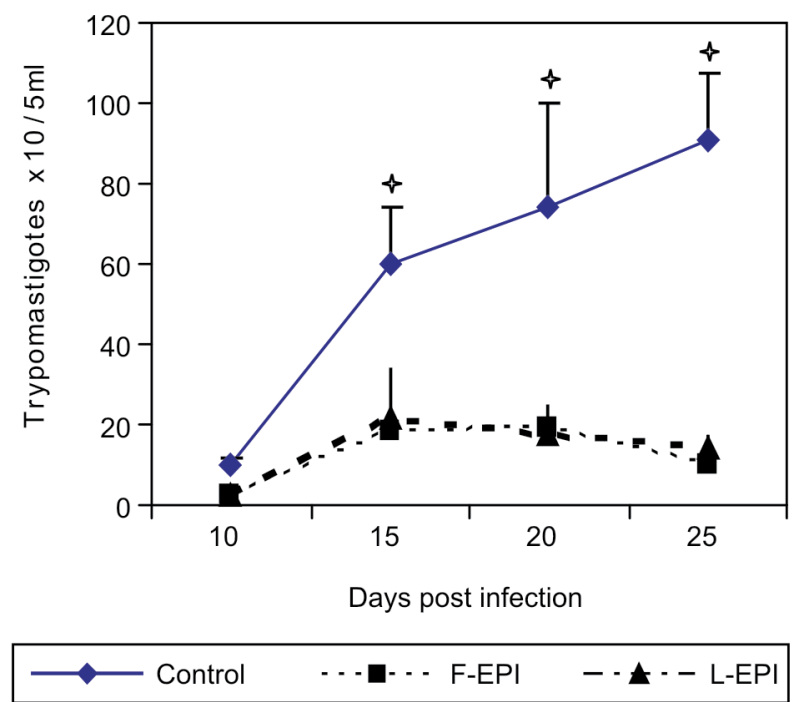

Fig. 1: levels of parasitemia in mice vaccinated with the Choachi strain of $T$. rangeli (F-EPI and L-EPI) and unvaccinated (control mice). Each time point represents the mean \pm SEM of 3-4 mice of a representative experiment. Asterisks indicate significance differences between vaccinated and infected groups (Student $t$ test, $\mathrm{p}<0.01$ ). day pi, there were no differences in parasitemia levels developed by the experimental and control groups. On the 20th day, both the L-EPI and F-EPI vaccinated groups presented significantly lower parasitemia levels than the control group $(p<0.01)$. This behavior was also observed at the 25th day pi. Similar results were observed with $T$. rangeli SC-58 strain (Fig. 2). Also in this case, the parasitemia levels of the vaccinated groups were lower than those in the control group $(\mathrm{p}<0.01)$.

More than $90 \%$ of the mice vaccinated with both strains of T. rangeli, live or fixed, survived after being challenged with T. cruzi. At day $30 \mathrm{pi}$, the parasites were no longer detected in the circulation of the vaccinated mice. In contrast, $62-75 \%$ of the animals of the control group died within 30 days pi. A representative experiment performed with F-EPI of Choachi strain can be seen in Fig. 3.

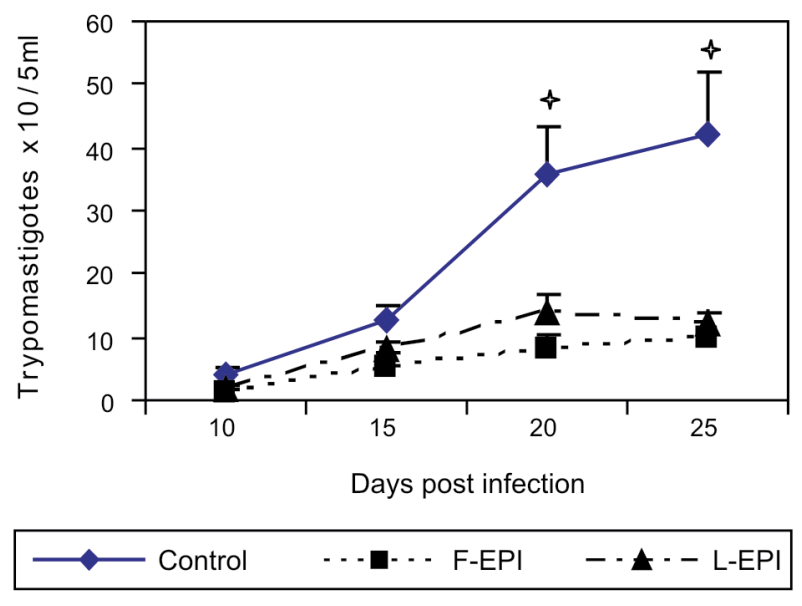

Fig. 2: levels of parasitemia in mice vaccinated with the SC 58 strain of . rangeli (F-EPI and L-EPI) and unvaccinated (control mice). Each time point represents the mean \pm SEM of 3-4 mice of a representative experiment. Asterisks indicate significant differences between vaccinated and infected groups (Student $t$ test $\mathrm{p}<0.01$ ).

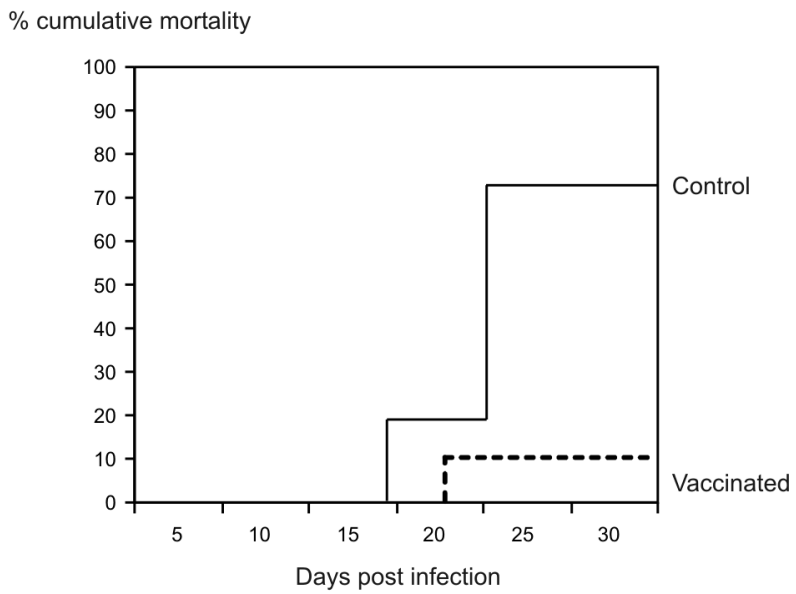

Fig. 3: cumulative mortality in mice vaccinated with F-EPI of Choachi strain of T. rangeli and in unvaccinated control mice infected with $T$. cruzi. 
Histological analysis of the hearts of the control mice revealed the typical histopathological alterations compatible with acute chagasic myocarditis, with numerous nests of amastigotes of $T$. cruzi and numerous lymphomononuclear inflammatory infiltrates of 10 or more cells (Fig. 4A, B). In comparison, the histological studies of the hearts of some of the mice vaccinated with the L-EPI $T$. rangeli SC-58 strain revealed myocarditis in the subendocardia, myocardia and epicardia, with zones of lymphomononuclear cells infiltration, but no amastigote nest (Fig. 4C, D). The skeletal muscle samples of the control mice exhibited disorganization of the tissue architecture, necrosis, and large nests of T. cruzi amastigotes (Fig. 5A B), while in the vaccinated mice, the skeletal muscle showed inflammatory infiltrates which consisted mainly of mononuclear cells. Again, no amastigote nest could be detected (Fig. 5C, D). Similar results were observed in the animals vaccinated with L-EPI of Choachi strain. In all mice vaccinated with F-EPI of both strains, the inflammatory infiltrates were milder in the heart and skeletal muscle samples.

The spleens of the mice immunized with live or fixed T. rangeli of both strains displayed an increased T-cell area, and also revealed a dramatically increased weight with respect to the control group. The weight ratio was expressed as spleen weight in $\mathrm{mg} /$ total body weight in grams. For the SC-58 strain, the results were: F-EPI: $22.09 \pm 0.80$; L-EPI: $19.44 \pm 2.33$; controls: $7.83 \pm 0.42$ $(\mathrm{n}=3$ of each group; $\mathrm{p}<0.001)$.

Tissues from the surviving control animals (infected only with $T$. cruzi) sacrificed in the chronic phase showed linear infiltrates of mononuclear cells and fragmentation of cardiac muscle fibers. The skeletal muscle showed evidence of necrosis and foci of mononuclear cells infiltrates. While the majority of immunized mice were histologically normal, some of them showed mild cardiac alterations. In some cases, the skeletal tissue revealed discrete mononuclear cell infiltrates. No amastigotes were found in either the control or in the vaccinated mice (data not shown).

\section{DISCUSSION}

In previous studies (Basso et al. 1991, Cervetta et al. 2002, Basso et al. 2004), we demonstrated that immunization with fixed epimastigotes of $T$. rangeli elicited Band T-cell-specific responses to T. cruzi, as well as a particular pattern of cytokines and a strong reduction in the mortality rate among infected mice. Indeed, more than $95 \%$ of the mice survived a lethal T. cruzi infection and displayed a strongly reduced burden of parasites during the acute phase; this was associated with high levels of antibodies and cellular response, elevated serum levels of IL-12 and IFN $\gamma$, low levels of pro-inflammatory cy-
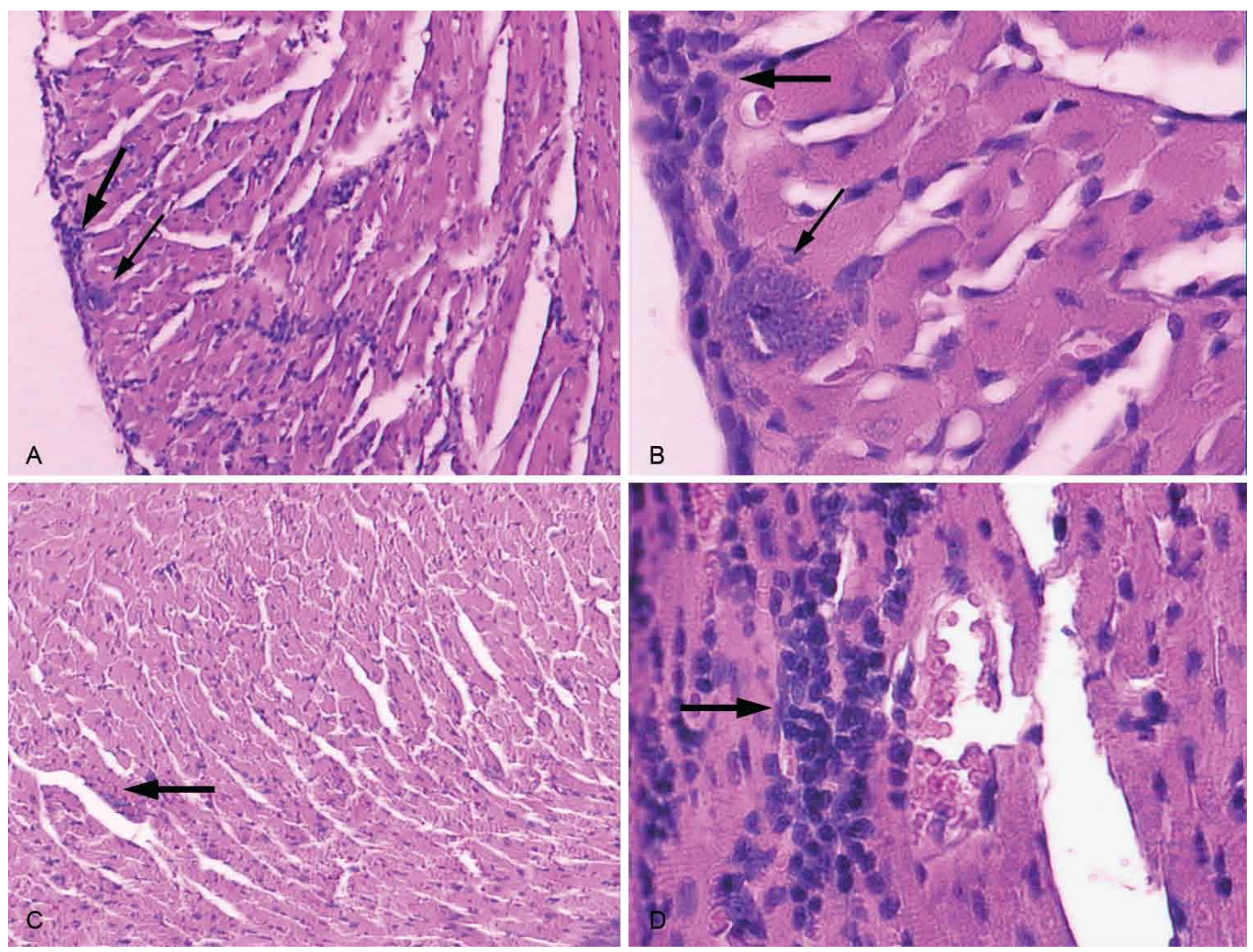

Fig. 4: histological sections of heart. A, B: control groups show nests of amastigotes (thin arrows) and mononuclear cell infiltration (thick arrow); C, D: representative sections from mice vaccinated with $T$. rangeli L-EPI of SC-58 strain. Infected mice show focal mononuclear cell infiltrates (thick arrows). No amastigote nest was observed (A, C: 100X; B, D: 400X). 


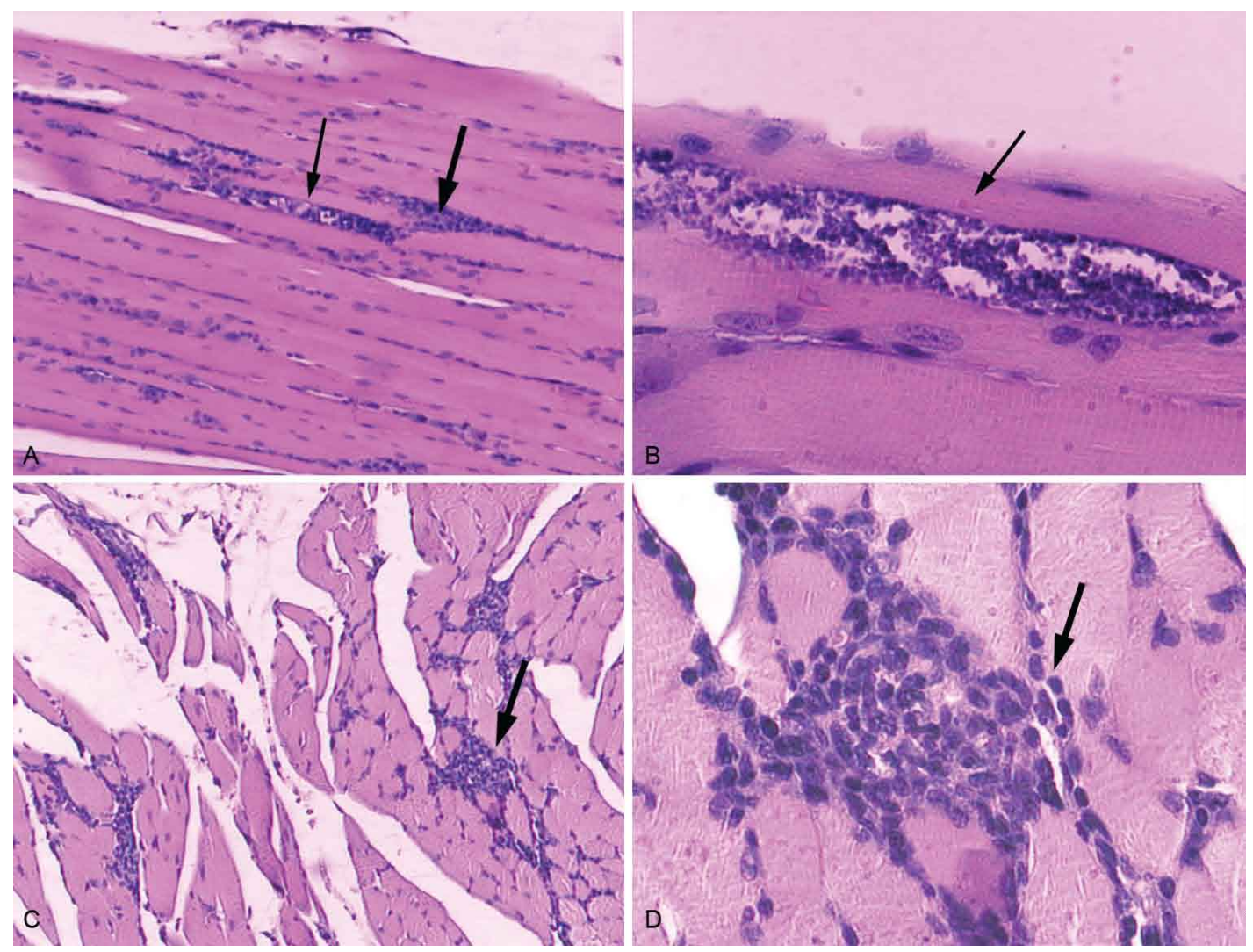

Fig. 5: histological sections of skeletal muscles. A, B: control groups show nests of amastigotes (thin arrows) and mononuclear cells infiltration (thick arrow); C, D: representative sections from mice vaccinated with L-EPI of $T$. rangeli SC-58 strain. Infected mice show focal mononuclear cell infiltrates (thick arrows). No amastigote nest was observed (A, C: 100X; B, D: 400X).

tokines (IL-6 and TNF $\alpha$ ) and normal levels of IL-10. The immunization induced an adequate balance of Th1 and Th2 responses, which are both necessary for the induction of a protective response.

In our present work, we demonstrated that the protection elicited by $T$. rangeli is a common feature displayed by at least three strains of $T$. rangeli of different geographical origins. In fact, in this study, we observed that immunization with epimastigotes of both the Brazilian strains of $T$. rangeli $\mathrm{SC}-58$ as well as the Colombian Choachi strain is sufficient to protect mice against a virulent $T$. cruzi strain, as was demonstrated with another Colombian strains (Basso et al. 1991). The vaccinated mice displayed low parasitemia, increased survival rate, absence of tissue amastigote nests in the acute period and an absence of histopathological lesions in the chronic period. The fact that this protection could be induced by strains of different origins is important for future vaccination strategies. These results are in accord with those reported by Zuñiga et al. (1997) and Palau et al. (2003). Moreover, using another experimental model, Araujo et al. (1999) have demonstrated that vaccination of $\mathrm{BALB} / \mathrm{c}$ mice with a combination of killed Leishmania promastigotes with BCG before challenging with $T$. cruzi prolonged their survival rate and decreased their parasitemia levels. It is interesting to note that the level of protection in mice depended on the number of T. rangeli used for immunization. In fact, as has been demonstrated in previous work (Cervetta et al. 2003), the minimum protective doses were $10^{7} /$ mouse with L-EPI and $10^{8} /$ mouse with F-EPI.

Although both immunogens (L-EPI and F-EPI) were able to induce protection, we assume that the use of live parasites in future human vaccines will be restricted due to the increased number of immunocompromised patients. Moreover, in our work, we have observed differences in histological studies that have revealed increased inflammatory infiltrates in animals vaccinated with live parasites. Taking these results into account, we have decided to continue our research on vaccination employing dead parasites.

At present, it is not known if infection with $T$. rangeli may change the course of infection with $T$. cruzi in humans. This does not exclude the possibility that an exposure to $T$. rangeli may elicit a particular humoral and/ or cellular immune response that confers some degree of protection against subsequent infection with $T$. cruzi (Palau et al. 2003, Caballero et al. 2007)

In conclusion, the results of the present work show that the antigens involved in the protection induced by T. rangeli are expressed in different strains of this parasite; this could be an advantage for developing future vaccination strategies for Chagas disease. In this sense, it will be also useful to advance in the identification of those protective antigens in order to use purified or synthesized molecules of $T$. rangeli in protection assays. 


\section{ACKNOWLEDGMENTS}

To Dr. Mario Steindel, from Laboratório de Protozoologia, Univ. Fed. Sta. Catarina, Brazil, for kindly providing us with the strains of $T$. rangeli employed in the present work, and Mrs. María Fernanda Garstein for editing and sharpening the translation to prepare the manuscript for publication.

\section{REFERENCES}

Araujo Z, El Bouhdidi A, Heremans H, Van Marck E, Castes M, Carlier Y 1999. Vaccination of mice with a combination of BCG and killed Leishmania promastigotes reduces acute Trypanosoma cruzi infection by promoting an IFN-gamma response. Vaccine 17: 957-964.

Basso B, Cervetta L, Moretti E, Carlier Y, Truyens C 2004. Acute Trypanosoma cruzi infection: IL-12, IL-18, TNF, sTNFR and NO in T. rangeli-vaccinated mice. Vaccine 22: 1868-1872.

Basso B, Moretti E, Vottero-Cima E 1987. Antigenic relationships between Trypanosoma cruzi and Trypanosoma rangeli. Rev Iber Parasitol 47: 15-21.

Basso B, Moretti ER, Vottero-Cima E 1991. Immune response and Trypanosoma cruzi infection in Trypanosoma rangeli- immunized mice. Am J Trop Med Hyg 44: 413-419.

Caballero ZC, Sousa OE, Marques WP, Saez-Alquezar A, Umezawa ES 2007. Evaluation of serological tests to identify Trypanosoma cruzi infection in humans and determine cross reactivity with Trypanosoma rangeli and Leishmania spp. Clin Vaccine Immunol 14: 1045-1049.

Cervetta L, Moretti E, Fretes R, Basso B 2003. Vaccination with live epimastigotes of $T$. rangeli protects mice against Trypanosoma cruzi lethal infection. Rev Patol Trop 32: 1-11.

Cervetta L, Moretti ER, Basso B 2002. Experimental Chagas' disease: the protection induced by immunization with Trypanosoma rangeli is associated with down-regulation of IL-6, TNFa and IL10 synthesis. Acta Parasitol 47: 73-78.

Costa F, Franchin G, Pereira-Chioccola VL, Ribeirao M, Schenkman $\mathrm{S}$, Rodrigues MM 1998. Immunization with a plasmid DNA containing the gene of transialidase reduces Trypanosoma cruzi infection in mice. Vaccine 16: 768-774.
Coura, JR 2007. Chagas disease: what is known and what is needed A background article. Mem Inst Oswaldo Cruz 102: 113-122.

Garg N, Tarleton RL 2002. Genetic immunization elicits antigen-specific protective immune responses and decreases disease severity in Trypanosoma cruzi infection. Infect Immun 70: 5547-5555.

Guhl F, Vallejo GA 2003. Trypanosoma (Herpetosoma) rangeli Tejera, 1920: an update review. Mem Inst Oswaldo Cruz 98: 435-442.

Hoff RA 1974. Methods of counting and concentrating living T. cruzi in blood lysed with ammonium chloride. J Parasitol 60: 527-528.

ILAR - Institute of Laboratory Animal Resources 1996. Guide for the care use of laboratory animals. National Research Council, National Academy Press, Washington, DC.

Introini MV, Basso B, Moretti ER 1998. Experimental Chagas disease: I. Study of different immunization conditions in the infection course. Bol Chil Parasitol 53: 45-51.

Palau MT, Mejia AJ, Vergara U, Zuniga CA 2003. Action of Trypanosoma rangeli in infections with virulent Trypanosoma cruzi populations. Mem Inst Oswaldo Cruz 98: 543-548.

Planelles L, Thomas MC, Alonso C, Lopez MC 2001. DNA immunization with Trypanosoma cruzi HSP70 fused to the KMP11 protein elicits a cytotoxic and humoral immune response against the antigen and leads to protection. Infect Immun 69: 6558-6563.

Stevens JR, Teixeira MM, Bingle LE, Gibson WC 1999. The taxonomic position and evolutionary relationships of Trypanosoma rangeli. Int J Parasitol 29: 749-757.

Taibi A, Espinoza AG, Ouaissi A 1995. Trypanosoma cruzi: analysis of cellular and humoral response against a protective recombinant antigen during experimental Chagas' disease. Immunol Lett 48: 193-200.

Wrightsman RA, Manning JE 2000. Paraflagellar rod proteins administered with alum and IL-12 or recombinant adenovirus expressing IL-12 generates antigen-specific responses and protective immunity in mice against Trypanosoma cruzi. Vaccine 18: 1419-1427.

Zuniga CA, Palau T, Penin P, Gramallo C, de Diego J 1997. Protective effect of Trypanosoma rangeli against infections with a highly virulent strain of Trypanosoma cruzi. Trop Med Int Health 2: 482-487. 